\title{
Thin-wall approximation in vacuum decay: A lemma
}

\author{
Adam R. Brown* \\ Physics Department, Stanford University, Stanford, California 94305, USA
}

(Received 16 February 2018; published 1 May 2018)

\begin{abstract}
The "thin-wall approximation" gives a simple estimate of the decay rate of an unstable quantum field. Unfortunately, the approximation is uncontrolled. In this paper I show that there are actually two different thin-wall approximations and that they bracket the true decay rate: I prove that one is an upper bound and the other a lower bound. In the thin-wall limit, the two approximations converge. In the presence of gravity, a generalization of this lemma provides a simple sufficient condition for nonperturbative vacuum instability.
\end{abstract}

DOI: $10.1103 /$ PhysRevD.97.105002

Metastable states may decay by quantum tunneling. In the semiclassical (small $\hbar$ ) limit, the most important contribution to the decay rate

$$
\text { rate } \sim A \exp [-B / \hbar]
$$

is the tunneling exponent $B$. In a famous paper Coleman explained how to calculate $B$ for a scalar field [1]. He showed that $B$ is given by the Euclidean action of an instanton that interpolates from the metastable (or "false") vacuum toward the target (or "true") vacuum. For a given field potential $V(\phi)$, the instanton can be derived by numerically integrating the second-order Euclidean equations of motion.

For those who lack either the computing power or the patience to solve the equations of motion, or who seek an intuitive understanding of the parametric dependence of the decay rate, Coleman also showed that we can often use the "thin-wall" approximation. While the instanton is a bubble that smoothly interpolates from the false vacuum to near the true vacuum, the thin-wall approximation treats this transition as abrupt [1]; the decay exponent is then approximated by

$$
B \sim \bar{B}_{\text {tw }} \equiv \frac{27 \pi^{2}}{2} \frac{\sigma^{4}}{\left(V_{\text {false }}-V_{\text {true }}\right)^{3}} .
$$

Consider these two expressions for the tension of the bubble wall $\sigma$,

\footnotetext{
*adambro@stanford.edu
}

Published by the American Physical Society under the terms of the Creative Commons Attribution 4.0 International license. Further distribution of this work must maintain attribution to the author(s) and the published article's title, journal citation, and DOI. Funded by SCOAP ${ }^{3}$.

$$
\begin{gathered}
\sigma_{\min } \equiv \int_{\phi_{*}}^{\phi_{\mathrm{f}}} d \phi \sqrt{2\left(V[\phi]-V\left[\phi_{\mathrm{f}}\right]\right)} ; \\
\sigma_{\max } \equiv \int_{\phi_{\mathrm{t}}}^{\phi_{\mathrm{f}}} d \phi \sqrt{2\left(V[\phi]-V\left[\phi_{\mathrm{t}}\right]\right)} .
\end{gathered}
$$

Coleman used only $\sigma_{\min }$ and left Eq. (2) as an uncontrolled approximation, in the sense that we are provided neither with an estimate of its accuracy nor with a bound on its error. We can do better. The main result of this paper is that, for any potential $V(\phi)$,

Lemma 1 (no gravity): $\bar{B}_{\mathrm{tw}}\left[\sigma_{\min }\right] \leq B \leq \bar{B}_{\mathrm{tw}}\left[\sigma_{\max }\right]$.

The first inequality is proved in Appendix A 1; the second in Appendix A2. As $V_{\text {false }}-V_{\text {true }} \rightarrow 0$ so too $\sigma_{\max }-$ $\sigma_{\min } \rightarrow 0$ and the two thin-wall approximations converge.

\begin{tabular}{lcc} 
& $G V_{\text {false }} \leq 0$ & $G V_{\text {false }}>0$ \\
\hline always $B \leq \bar{B}_{\mathrm{tw}}^{G}\left[\sigma_{\text {max }}\right] ?$ & PROVED & OPEN \\
always $B \geq \bar{B}_{\mathrm{tw}}^{G}\left[\sigma_{\text {max }}\right]$ ? & FALSE [5] & FALSE [4] \\
\hline
\end{tabular}

We can partially generalize this to include gravity. As first calculated by Coleman and de Luccia [2], gravitational backreaction changes $B$ : the decay exponent now depends not just on the difference $V_{\text {false }}-V_{\text {true }}$, but also on $V_{\text {false }}$ and $\mathrm{V}_{\text {true }}$ separately, since zero-point energy curves spacetime. The gravitational generalization of the thin-wall approximation to the decay exponent, $\bar{B}_{\mathrm{tw}}^{G}[\sigma]$, is given by Eq. (B5), and the partial generalization of Lemma 1 is that

Lemma2

$$
\text { (when } \left.G V_{\text {false }} \leq 0\right): B \leq \bar{B}_{\mathrm{tw}}^{G}\left[\sigma_{\mathrm{max}}\right] \text {. }
$$

This inequality is proved in Appendix B. The addition of gravity means it is no longer always true that $B \geq \bar{B}_{\mathrm{tw}}^{G}\left[\sigma_{\mathrm{min}}\right]$ : a de Sitter false vacuum $\left(G V_{\text {false }}>0\right)$ with a short but very 

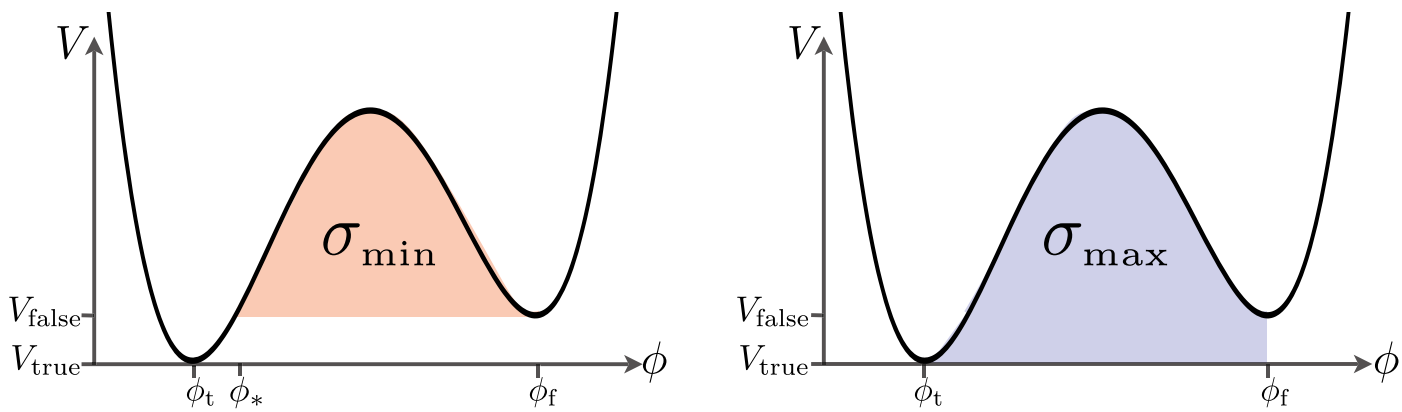

FIG. 1. The field starts in the false vacuum $\phi_{\mathrm{f}}$ and tunnels towards the true vacuum $\phi_{\mathrm{t}}$. The definition of $\phi_{*}$ is such that $V\left[\phi_{*}\right]=V\left[\phi_{\mathrm{f}}\right]$. Equation (3) gives two different definitions of the tension, with $\sigma_{\max }>\sigma_{\min }$.

broad barrier has arbitrarily large $\sigma_{\min }$ but still decays relatively promptly via a Hawking-Moss instanton [3], so that $B \ll \bar{B}_{\mathrm{tw}}^{G}\left[\sigma_{\min }\right]<\bar{B}_{\mathrm{tw}}^{G}\left[\sigma_{\max }\right]$; and a Minkowski false vacuum $\left(G V_{\text {false }}=0\right)$ that has $B<\bar{B}_{\mathrm{tw}}^{G}\left[\sigma_{\mathrm{min}}\right]$ was exhibited numerically in Sec. V of [4]. (It is still open whether $B$ may be greater than $\bar{B}_{\mathrm{tw}}^{G}\left[\sigma_{\max }\right]$ for $G V_{\text {false }}>0$; I suspect it may not.)

One application of these lemmas is diagnosing instability. Gravity can stabilize superficially metastable $G V_{\text {false }} \leq 0$ vacua [2], and there is great interest in determining which vacua decay and which endure (see e.g. [5]). Lemma 2 provides a sufficient condition for instability, which is that $\bar{B}_{\mathrm{tw}}^{G}\left[\sigma_{\max }\right]<\infty$, or equivalently

sufficient condition for instability:

$$
\sigma_{\max }<\frac{\sqrt{-V\left[\phi_{\text {true }}\right]}-\sqrt{-V\left[\phi_{\text {false }}\right]}}{\sqrt{6 \pi G}} .
$$

No such condition is required for $G V_{\text {false }}>0$, since all de Sitter false vacua are unstable [2].

We can generalize Lemma 2 by replacing $\phi_{\mathrm{t}}$ with any value in the range $\phi_{\mathrm{t}} \leq \phi_{\text {mid }}<\phi_{*}$, as shown in Fig. 2. Thus the false vacuum is unstable if there exists any $\phi_{\text {mid }}$ such that

$$
\begin{aligned}
\sigma_{\text {mid }} \equiv & \int_{\phi_{\text {mid }}}^{\phi_{\mathrm{f}}} d \phi \sqrt{2\left(V[\phi]-V\left[\phi_{\text {mid }}\right]\right)} \\
& <\frac{\sqrt{-V\left[\phi_{\text {mid }}\right]}-\sqrt{-V\left[\phi_{\text {false }}\right]}}{\sqrt{6 \pi G}} .
\end{aligned}
$$

When there are multiple fields, there are many possible routes over the barrier, and this condition applies to all of them: if we can find any route for the $\sigma_{\text {mid }}$ integral such that Eq. (7) holds, the vacuum must be unstable, the field must eventually decay, and spacetime is doomed [2].

\section{ACKNOWLEDGMENTS}

Thank you to Sonia Paban and Erick Weinberg for reading a draft of this paper, and to the organizers of TASI '09 for their hospitality while these lemmas were established.

\section{APPENDIX A: PROVING NONGRAVITATIONAL RESULTS}

This appendix proves Lemma 1 . The two inequalities demand two very different proof strategies.

Consider the decay of the false vacuum of Fig. 1. We will assume that there are no intervening minima between the false and true vacua, but otherwise leave the potential completely general. I will now review how to calculate the vacuum decay rate; this is all explained with great clarity in [1]. In [1] it is shown that the tunneling exponent is given by the Euclidean action of an instanton. The instanton $\bar{\phi}(\tau, \vec{x})$ lives on $\mathbb{R}^{4}$ and extremizes the Euclidean action

$$
\begin{aligned}
S_{E} & =\int d^{4} x\left(\frac{1}{2}\left(\partial_{\mu} \phi\right)^{2}+V(\phi)\right) \\
& =2 \pi^{2} \int d \rho \rho^{3}\left(\frac{1}{2} \dot{\phi}^{2}+V(\phi)\right),
\end{aligned}
$$

where we have written $d s^{2}=d \tau^{2}+d \vec{x}^{2}=d \rho^{2}+\rho^{2} d \Omega_{3}^{2}$ and used that the instanton can be shown [6] to have $\mathrm{O}(4)$ spherical symmetry $\bar{\phi}=\bar{\phi}(\rho)$. One boundary condition is that the field returns to the false vacuum $\bar{\phi} \rightarrow \phi_{\mathrm{f}}$ as $\rho \rightarrow \infty$; the other boundary condition is that the field immediately after nucleation, given by

field after nucleation: $\phi(t=0, \vec{x})=\bar{\phi}(\tau=0, \vec{x})$,

has the same energy as the false vacuum $(\Delta E=0)$ and classically evolves toward the true vacuum. (In fact, the symmetry tells us that it will give rise to a bubble of approximately true vacuum that expands out at approaching the speed of light.) The instanton gives a path through the space of $\phi(\vec{x})$ s that connects the before-tunneling configuration $\phi(\tau=-\infty, \vec{x})=\phi_{\mathrm{f}}$ to the after-tunneling configuration $\phi(\tau=0, \vec{x})$. Indeed, the instanton is defined as the solution of minimum Euclidean action that satisfies these boundary conditions - in the language of [7], it is the most probable decay path. ${ }^{1}$

\footnotetext{
${ }^{1}$ Technically we insist that the decay path ends at its first intersection with the $\Delta E=0$ surface.
} 

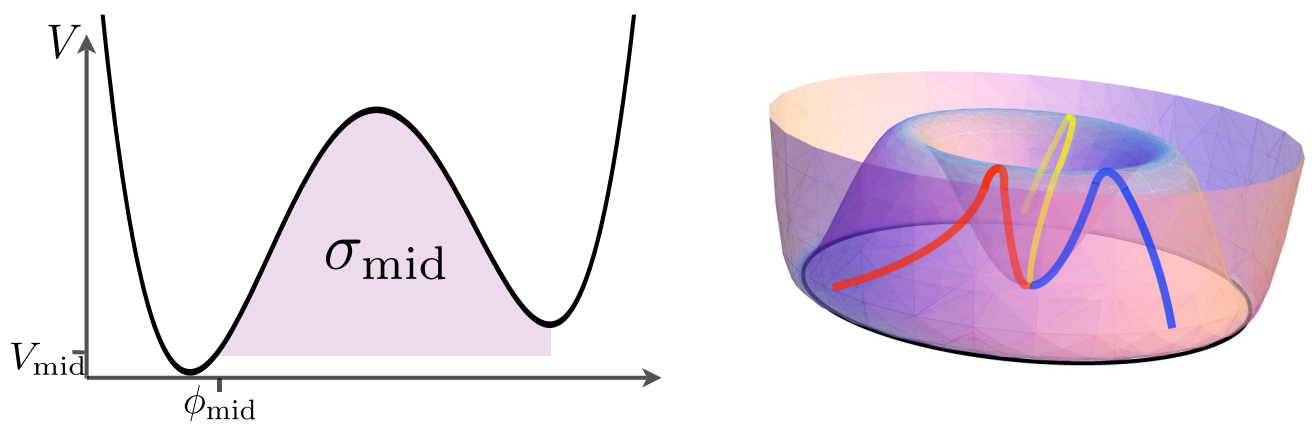

FIG. 2. If we can find any path across the barrier that satisfies Eq. (7), the vacuum must be unstable. Left: the $\sigma$ integral need not be taken all the way to $\phi_{\text {true }}$, and can instead stop anywhere in the range $\phi_{\text {true }} \leq \phi_{\text {mid }}<\phi_{*}$. Right: when there are multiple fields, there are many escape routes, and the decay path may take any of them.

(With no constraint on the energy of the $\tau=0$ configuration, the instanton has exactly one negative mode [8]; this negative mode is associated with changing the energy of the nucleated bubble away from $\Delta E=0$. In this paper we will freeze this negative mode by only considering paths that end with the same energy as the false vacuum $\Delta E=0$; amongst this set of paths the instanton is a minimum of the Euclidean action [9].)

To minimize the action, the instanton must satisfy the Euler-Lagrange equation,

$$
\frac{d}{d \rho}\left(\frac{1}{2} \dot{\bar{\phi}}^{2}-V(\bar{\phi})\right)=-\frac{3}{\rho} \dot{\bar{\phi}}^{2}
$$

This equation implies that $\frac{1}{2} \dot{\bar{\phi}}^{2}-V(\bar{\phi})$ monotonically decreases as $\rho$ increases (in [1] this is called "friction"), which in turn implies that

$$
\begin{gathered}
-V_{\text {false }}<\frac{1}{2} \dot{\bar{\phi}}^{2}-V(\phi)<-V_{\text {true }} \\
\rightarrow \sqrt{2\left(V-V_{\text {false }}\right)}<\dot{\bar{\phi}}<\sqrt{2\left(V-V_{\text {true }}\right)} .
\end{gathered}
$$

The tunneling exponent is then given by the difference in Euclidean action between the instanton and the false vacuum

$$
B=S_{E}(\bar{\phi})-S_{E}\left(\bar{\phi}_{\mathrm{f}}\right)=2 \pi^{2} \int_{0}^{\infty} d \rho \rho^{3}\left(\frac{1}{2} \dot{\phi}^{2}+V(\phi)-V_{\text {false }}\right) .
$$

Since tunneling conserves energy, immediately after nucleation the bubble must have the same energy as the false vacuum,

$\Delta E \equiv 4 \pi \int_{0}^{\infty} d \rho \rho^{2}\left(\frac{1}{2} \dot{\bar{\phi}}^{2}+V(\bar{\phi})-V_{\text {false }}\right)=0$.

But the energy density is not zero everywhere. Instead, the energy density is positive in the "wall" of the bubble where the field traverses the barrier, and then negative inside the bubble. Fig. 3 shows a cross section of a typical bubble. "Outside" the bubble $\left(\phi>\phi_{*}\right)$ the energy density is bigger than $V_{\text {false }}$; "inside" the bubble $\left(\phi<\phi_{*}\right)$ we know only that the energy density is bigger than $V_{\text {true }}$.

Consider the field value at the very center of the bubble, $\phi(\rho=0)$. It follows from the conservation of energy that $\phi(0) \leq \phi_{*}$; conversely it follows from Eq. (A5) that $\phi(0) \geq \phi_{\mathrm{t}}$. Indeed, the fact that $\phi_{\mathrm{t}} \leq \phi(0) \leq \phi_{*}$ is what originally motivated the definitions of $\sigma_{\min }$ and $\sigma_{\max }$ in Eq. (3): the two lower limits of integration capture the full range of possible values of $\phi(0)$.

For the remainder of Appendix A we will add a constant to the potential to set $V_{\text {false }}=0$.

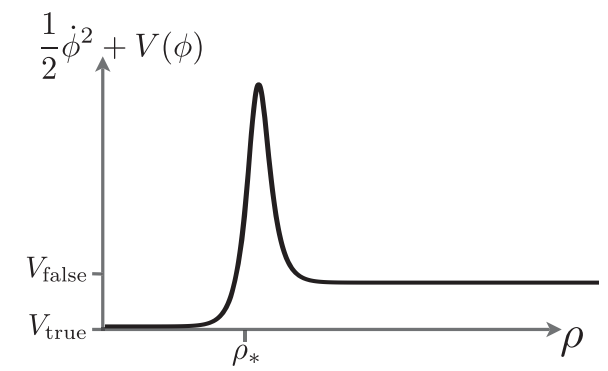

FIG. 3. A cross-section through a typical bubble at the moment of nucleation. The center of the bubble may have an energy density as low as $V_{\text {true }}$; but for $\rho>\rho_{*}$ the energy density necessarily exceeds that of the false vacuum $\frac{1}{2} \dot{\phi}^{2}+V(\phi) \geq V(\phi) \geq V_{\text {false }}$. The total integrated energy is equal to that in the false vacuum, $\Delta E=0$. 

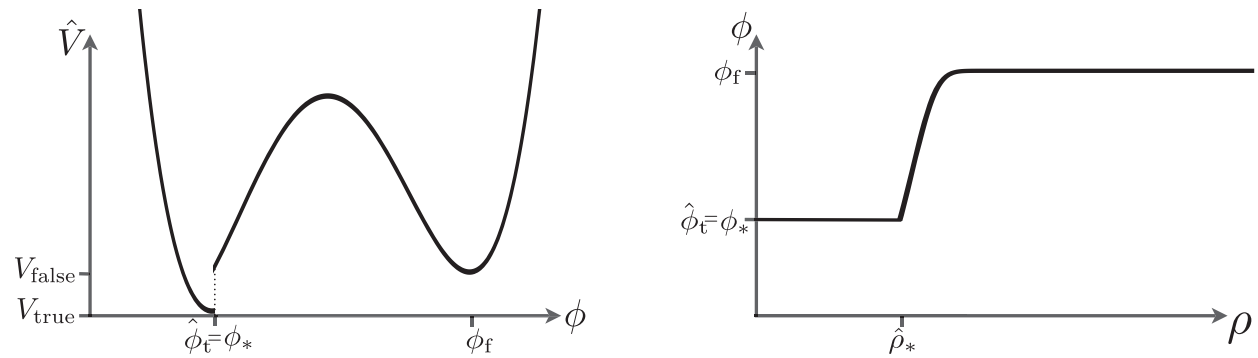

FIG. 4. $\hat{V}[\phi]$ is constructed by deleting the part of $V(\phi)$ that lies between $\phi_{\mathrm{t}}$ and $\phi_{*}$. The corresponding bubble instanton has pure true vacuum inside some radius that we will call $\hat{\rho}_{*}$. The bubble has energy $\Delta \hat{E}=0$.

\section{Proving $B \geq \bar{B}_{\mathrm{tw}}\left[\boldsymbol{\sigma}_{\min }\right]$}

Proof strategy: The instanton is a bubble configuration with zero energy, $\Delta E=0$. In order to have $\Delta E=0$, any bubble must be big, and if it is big enough it must have $B \geq \bar{B}_{\mathrm{tw}}\left[\sigma_{\mathrm{min}}\right]$.

First let's construct a new potential $\hat{V}[\phi]$ that decays faster than $V[\phi]$,

$$
\hat{V}[\phi] \equiv \begin{cases}V[\phi] & \text { for } \phi>\phi_{*} \\ V\left[\phi-\phi_{*}+\phi_{\mathrm{t}}\right] & \text { for } \phi<\phi_{*}\end{cases}
$$

The region of $V[\phi]$ between $\phi_{\mathrm{t}}$ and $\phi_{*}$ has been excised, with the $\phi>\phi_{*}$ part glued straight onto the $\phi<\phi_{\mathrm{t}}$ part. This does not affect $\sigma_{\min }$ or $V_{\text {false }}$ or $V_{\text {true }}$, but since we have removed part of the barrier the decay rate is faster

$$
B[V(\phi)] \geq \hat{B} \equiv B[\hat{V}(\phi)] .
$$

The shape of the bubble is plotted in Fig. 4. The bubble stays uniformly in the true vacuum until $\rho=\hat{\rho}_{*}$, and then proceeds towards the false vacuum with a profile given by Eq. (A3).

Let us calculate $\hat{B}$. First notice that by changing variables from $\rho$ to $\phi$,

$$
\begin{aligned}
\int_{\hat{\rho}_{*}}^{\infty} d \rho\left(\frac{1}{2} \dot{\bar{\phi}}^{2}+V[\bar{\phi}]\right) \\
=\int_{\phi_{*}}^{\phi_{\mathrm{f}}} \frac{d \phi}{\dot{\bar{\phi}}}\left(\frac{1}{2} \dot{\bar{\phi}}^{2}+V[\bar{\phi}]\right) \\
=\int_{\phi_{*}}^{\phi_{\mathrm{f}}} d \phi \frac{\frac{1}{2}(\dot{\bar{\phi}}-\sqrt{2 V})^{2}+\sqrt{2 V} \dot{\bar{\phi}}}{\dot{\bar{\phi}}} \geq \sigma_{\min } .
\end{aligned}
$$

The total energy of the bubble, relative to the false vacuum, is

$$
\begin{aligned}
\Delta \hat{E}= & 4 \pi \int_{0}^{\hat{\rho}_{*}} d \rho \rho^{2}\left(\frac{1}{2} \dot{\bar{\phi}}^{2}+V[\bar{\phi}]\right) \\
& +4 \pi \int_{\hat{\rho}_{*}}^{\infty} d \rho \rho^{2}\left(\frac{1}{2} \dot{\bar{\phi}}^{2}+V[\bar{\phi}]\right)
\end{aligned}
$$

$$
\begin{aligned}
& \geq 4 \pi \int_{0}^{\hat{\rho}_{*}} d \rho \rho^{2} V_{\text {true }}+4 \pi \hat{\rho}_{*}^{2} \int_{\hat{\rho}_{*}}^{\infty} d \rho\left(\frac{1}{2} \dot{\bar{\phi}}^{2}+V[\bar{\phi}]\right) \\
& \geq \frac{4 \pi}{3} \hat{\rho}_{*}^{3} V_{\text {true }}+4 \pi \hat{\rho}_{*}^{2} \sigma_{\text {min }} .
\end{aligned}
$$

Tunneling conserves energy, $\Delta \hat{E}=0$, so the bubble must be large

$$
\hat{\rho}_{*} \geq \frac{3 \sigma_{\min }}{\left(-V_{\text {true }}\right)} .
$$

Since $\Delta \hat{E}=0$ implies $\hat{B}=\hat{B}-\frac{\pi \hat{\rho}_{*} \Delta \hat{E}}{2}$, we can use Eqs. (A6) and (A10) to prove our result

$$
\begin{aligned}
B \geq \hat{B} & =2 \pi^{2} \int_{0}^{\infty} d \rho \rho^{2}\left(\rho-\hat{\rho}_{*}\right)\left(\frac{1}{2} \dot{\bar{\phi}}^{2}+\hat{V}[\bar{\phi}]\right) \\
& \geq 2 \pi^{2} \int_{0}^{\hat{\rho}_{*}} d \rho \rho^{2}\left(\rho-\hat{\rho}_{*}\right)\left(\frac{1}{2} \dot{\bar{\phi}}^{2}+\hat{V}[\bar{\phi}]\right) \\
& \geq 2 \pi^{2} \int_{0}^{\hat{\rho}_{*}} d \rho \rho^{2}\left(\rho-\hat{\rho}_{*}\right) V_{\text {true }} \\
& \geq 2 \pi^{2} \frac{\hat{\rho}_{*}^{4}\left(-V_{\text {true }}\right)}{12} \\
& \geq \frac{27 \pi^{2}}{2} \frac{\sigma_{\text {min }}^{4}}{\left(-V_{\text {true }}\right)^{3}}=\bar{B}_{\text {tw }}\left[\sigma_{\text {min }}\right] .
\end{aligned}
$$

\section{Proving $B \leq \overline{\boldsymbol{B}}_{\mathrm{tw}}\left[\boldsymbol{\sigma}_{\max }\right]$}

Proof strategy: The instanton is the path of minimum action that interpolates from the false vacuum to a $\Delta E=0$ state on the true vacuum side of the barrier. I will explicitly construct an interpolating path with action $\bar{B}_{\mathrm{tw}}\left[\sigma_{\max }\right]$.

Consider the one-parameter family of field-profiles ${ }^{2}$ $\phi_{\bar{R}}(\rho)$ parameterized by $\bar{R}$ and defined by

\footnotetext{
${ }^{2}$ This field-profile has a discontinuous first derivative at both $\rho=\bar{R}$ and $\rho=0$. This indicates the field-profile is not a minimum of the Euclidean action, but does not prevent the field-profile from contributing to the path integral- to contribute to the path integral, a field-profile only needs to be continuous, not differentiable. If desired, my proof can be reformulated entirely in terms of differentiable field-profiles: smoothing the field at $\rho=0$ and $\rho=R$ will only make a tiny (second-order) change to the action, so the inequality would still follow.
} 


$$
\begin{gathered}
\phi_{\bar{R}}(\rho)=\phi_{\mathrm{f}} \quad \text { for } \rho>\bar{R} \\
\dot{\phi}_{\bar{R}}(\rho)=\sqrt{2\left(V\left[\phi_{\bar{R}}(\rho)\right]-V_{\text {true }}\right)} \text { for } \rho<\bar{R} .
\end{gathered}
$$

From the definition it follows that $\phi_{\mathrm{t}}<\phi_{\bar{R}}(\rho) \leq \phi_{\mathrm{f}}$. The Euclidean action of $\phi_{\bar{R}}(\rho)$ is

$$
\begin{aligned}
S_{E}[\bar{R}] & =2 \pi^{2} \int_{0}^{\bar{R}} d \rho \rho^{3}\left(\frac{1}{2} \dot{\phi}_{\bar{R}}^{2}+V\left[\phi_{\bar{R}}\right]\right) \\
& =2 \pi^{2} \int_{0}^{\bar{R}} d \rho \rho^{3}\left(2\left(V[\phi]-V_{\text {true }}\right)\right)+2 \pi^{2} \int_{0}^{\bar{R}} d \rho \rho^{3}\left(V_{\text {true }}\right) \\
& \leq 2 \pi^{2} \bar{R}^{3} \int_{\phi_{\bar{R}}[0]}^{\phi_{\mathrm{f}}} d \phi \sqrt{2\left(V[\phi]-V_{\text {true }}\right)}+\frac{2 \pi^{2}}{4} V_{\text {true }} \bar{R}^{4} \\
& \leq 2 \pi^{2} \bar{R}^{3} \sigma_{\max }-\frac{\pi^{2}}{2}\left(-V_{\text {true }}\right) \bar{R}^{4} \\
& \leq \frac{27 \pi^{2}}{2} \frac{\sigma_{\max }^{4}}{\left(-V_{\text {true }}\right)^{3}}=\bar{B}_{\text {tw }}\left[\sigma_{\text {max }}\right] .
\end{aligned}
$$

Our family of field-profiles $\phi_{\bar{R}}(\rho)$ must contain as "escape path," in the language of [7]: while small values of $\bar{R}$ gives positive energy $\Delta E>0$, arbitrarily large values of $\bar{R}$ give arbitrarily negative energies, so there must be an intervening value $\bar{R}_{E=0}$ such that $\Delta E\left[\phi_{\bar{R}}(t=0, \vec{x})\right]=0$. Therefore the most probable escape path has $B \leq S_{E}\left[\bar{R}_{E=0}\right] \leq \bar{B}_{\mathrm{tw}}\left[\sigma_{\mathrm{max}}\right]$.

This proof would still have gone through had we replaced $V_{\text {true }}$ by $V_{\text {mid }} \equiv V\left[\phi_{\text {mid }}\right]$ in Eq. (A19), for any $\phi_{\text {mid }}$ in the range $\phi_{\mathrm{t}} \leq \phi_{\text {mid }}<\phi_{*}$. Thus a strengthening of the lemma is that

$$
\begin{aligned}
B & \leq \frac{27 \pi^{2}}{2} \frac{\sigma_{\text {mid }}^{4}}{\left(V_{\text {false }}-V_{\text {mid }}\right)^{3}}, \quad \text { where } \\
\sigma_{\text {mid }} & \equiv \int_{\phi_{\text {mid }}}^{\phi_{\mathrm{f}}} d \phi \sqrt{2\left(V[\phi]-V\left[\phi_{\text {mid }}\right]\right)}
\end{aligned}
$$

for every possible $\phi_{\text {mid }}$, and (for multifield potentials) for every possible route over the barrier.

\section{APPENDIX B: PROVING GRAVITATIONAL RESULT}

When gravity is included, the decaying field curves spacetime. Despite this complication, the same general proof strategy will apply as for the nongravitational case of Sec. A 2.

The formalism that governs the gravitational decay of the false vacuum was described with great clarity in [2]. With gravity included, it has not been proved that the dominant instanton is still $O(4)$-symmetric; following conventional wisdom, we will assume that it is. The metric may then be written

$$
d s^{2}=d \xi^{2}+\rho(\xi)^{2} d \Omega_{3}^{2} .
$$

Matter tells space how to curve

$$
\dot{\rho}^{2}=1+\frac{8 \pi G}{3} \rho^{2}\left(\frac{1}{2} \dot{\phi}^{2}-V(\phi)\right)
$$

and space tells matter how to move

$$
\frac{d}{d \xi}\left(\frac{1}{2} \dot{\bar{\phi}}^{2}-V(\bar{\phi})\right)=-3 \frac{\dot{\rho}(\xi)}{\rho(\xi)} \dot{\bar{\phi}}^{2}
$$

When the gravitational constraint Eq. (B2) is satisfied, the action is given by Eq. (3.9) of [2] as

$S_{E}=4 \pi^{2} \int d \xi\left(\rho^{3} V-\frac{3}{8 \pi G} \rho\right)=4 \pi^{2} \int d \rho \frac{\rho^{3} V-\frac{3}{8 \pi G} \rho}{\dot{\rho}}$.

The thin-wall approximation ${ }^{3}$ to the tunneling exponent $[2,14]$ is

$$
\begin{aligned}
\bar{B}_{\mathrm{tw}}^{G} \equiv & 2 \pi^{2} \bar{\rho}^{3} \sigma+\frac{3}{16} \frac{\left(1-\frac{8 \pi G}{3} \bar{\rho}^{2} V_{\text {true }}\right)^{\frac{3}{2}}-1}{G^{2} V_{\text {true }}} \\
& -\frac{3}{16} \frac{\left(1-\frac{8 \pi G}{3} \bar{\rho}^{2} V_{\text {false }}\right)^{\frac{3}{2}}-1}{G^{2} V_{\text {false }}},
\end{aligned}
$$

where $\bar{\rho}$ is the radius of the bubble wall that maximizes Eq. (B5), namely (for $G V_{f} \leq 0$ )

$$
\bar{\rho} \equiv \frac{3 \sigma}{\sqrt{\left(\sqrt{-V_{\mathrm{t}}}-\sqrt{-V_{\mathrm{f}}}\right)^{2}-6 \pi G \sigma^{2}} \sqrt{\left(\sqrt{-V_{\mathrm{t}}}+\sqrt{-V_{\mathrm{f}}}\right)^{2}-6 \pi G \sigma^{2}}} .
$$

\footnotetext{
${ }^{3}$ For numerical investigations of the reliability of the thin-wall approximation in the nongravitational case, see [10]; see also [11]. For numerical investigations in the gravitational case, see $[4,12]$; see also [13].
}

As $\sigma$ approaches $\left(\sqrt{-V_{\mathrm{t}}}-\sqrt{-V_{\mathrm{f}}}\right) / \sqrt{6 \pi G}$ both $\bar{\rho}$ and $\bar{B}_{\mathrm{tw}}^{G}$ diverge; for larger values of the tension, the thin-wall approximation predicts that the false vacuum is stable.

To prove the gravitational lemma [Eq. (5)] we will follow Sec. A 2 in constructing a family of paths and showing that one member of the family is an escape path, and every 

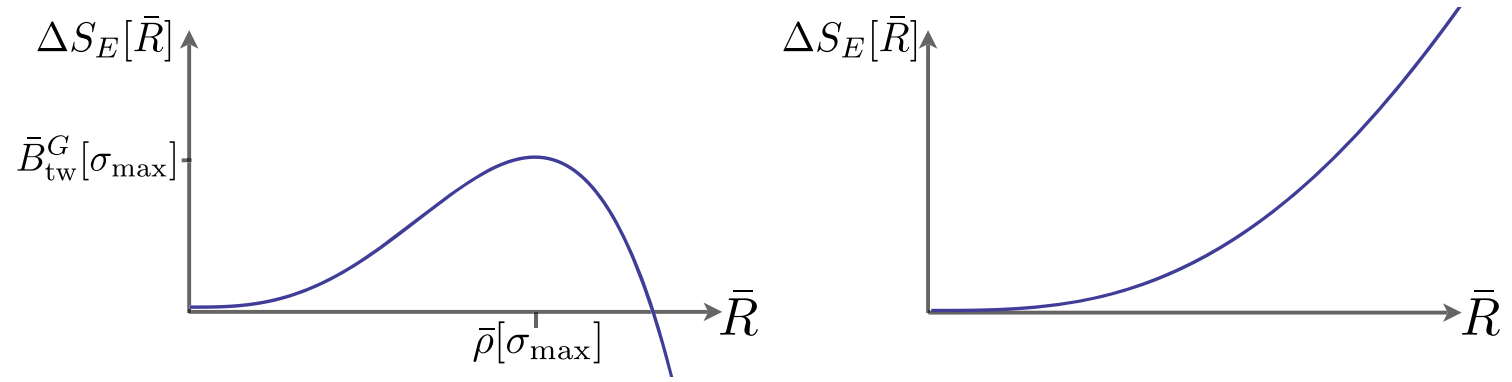

FIG. 5. Left: for $\sigma_{\max }<\left(\sqrt{-V_{\mathrm{t}}}-\sqrt{-V_{\mathrm{f}}}\right) / \sqrt{6 \pi G}$, the function $\Delta S_{E}[\bar{R}]$ from Eq. (B10) never exceeds $\bar{B}_{\mathrm{tw}}^{G}\left[\sigma_{\mathrm{max}}\right]$. Right: for $\sigma_{\max } \geq\left(\sqrt{-V_{\mathrm{t}}}-\sqrt{-V_{\mathrm{f}}}\right) / \sqrt{6 \pi G}$, the function $\Delta S_{E}[\bar{R}]$ may (or may not) grow without bound.

member of the family has $\Delta S_{E} \leq \bar{B}_{\mathrm{tw}}^{G}\left[\sigma_{\max }\right]$. The family of field-profiles will be parametrized by $\bar{R}$,

$$
\begin{aligned}
\phi_{\bar{R}}(\xi)=\phi_{\mathrm{f}} \rightarrow \dot{\rho}^{2} & =1-\frac{8 \pi G}{3} \rho^{2} V_{\text {false }} \quad \text { for } \rho>\bar{R} \\
\dot{\phi}_{\bar{R}}(\xi) & =\sqrt{2\left(V[\phi(\xi)]-V_{\text {true }}\right)} \\
\rightarrow \dot{\rho}^{2} & =1-\frac{8 \pi G}{3} \rho^{2} V_{\text {true }} \quad \text { for } \rho<\bar{R}
\end{aligned}
$$

Notice that while this family need not satisfy the equation of motion for the field, Eq. (B3), it is required to satisfy the gravitational constraint, Eq. (B2), because only such configurations contribute to the gravitational path integral. Notice also that $\dot{\rho} \geq 1>0$ so $\rho$ monotonically increases with $\xi$ and the topology is $\mathbb{R}^{4}$. The field and metric differ from the false vacuum only inside $\rho<\bar{R}$, so the difference in action $\Delta S_{E}=S_{E}\left[\phi_{\bar{R}}(\rho)\right]-S_{E}\left[\phi_{\mathrm{f}}\right]$ is

$$
\Delta S_{E}=4 \pi^{2} \int_{0}^{\bar{R}} d \rho\left(\frac{\rho^{3} V-\frac{3}{8 \pi G} \rho}{\sqrt{1-\frac{8 \pi G}{3} \rho^{2} V_{\text {true }}}}-\frac{\rho^{3} V_{\text {false }}-\frac{3}{8 \pi G} \rho}{\sqrt{1-\frac{8 \pi G}{3} \rho^{2} V_{\text {false }}}}\right)
$$

$$
\begin{aligned}
& =4 \pi^{2} \int_{0}^{\bar{R}} d \rho\left(\frac{\rho^{3}\left(V-V_{\text {true }}\right)}{\sqrt{1-\frac{8 \pi G}{3} \rho^{2} V_{\text {true }}}}+\frac{\rho^{3} V_{\text {true }}-\frac{3}{8 \pi G} \rho}{\sqrt{1-\frac{8 \pi G}{3} \rho^{2} V_{\text {true }}}}-\frac{\rho^{3} V_{\text {false }}-\frac{3}{8 \pi G} \rho}{\sqrt{1-\frac{8 \pi G}{3} \rho^{2} V_{\text {false }}}}\right) \\
& =4 \pi^{2} \int_{\phi_{\bar{R}}[0]}^{\phi_{\mathrm{f}}} \frac{d \phi \rho^{3}\left(V-V_{\mathrm{t}}\right)}{\sqrt{2\left(V-V_{\mathrm{t}}\right)}}+\frac{3}{16} \frac{\left(1-\frac{8 \pi G}{3} \bar{R}^{2} V_{\mathrm{t}}\right)^{\frac{3}{2}}-1}{G^{2} V_{\mathrm{t}}}-\frac{3}{16} \frac{\left(1-\frac{8 \pi G}{3} \bar{R}^{2} V_{\mathrm{f}}\right)^{\frac{3}{2}}-1}{G^{2} V_{\mathrm{f}}} \\
& \leq 2 \pi^{2} \bar{R}^{3} \sigma_{\max }+\frac{3}{16} \frac{\left(1-\frac{8 \pi G}{3} \bar{R}^{2} V_{\text {true }}\right)^{\frac{3}{2}}-1}{G^{2} V_{\text {true }}}-\frac{3}{16} \frac{\left(1-\frac{8 \pi G}{3} \bar{R}^{2} V_{\text {false }}\right)^{\frac{3}{2}}-1}{G^{2} V_{\text {false }}}
\end{aligned}
$$

$$
\leq \bar{B}_{\mathrm{tw}}^{G}\left[\sigma_{\max }\right] .
$$

There are two possible cases, both shown in Fig. 5. First consider $\sigma_{\max }<\left(\sqrt{-V_{\mathrm{t}}}-\sqrt{-V_{\mathrm{f}}}\right) / \sqrt{6 \pi G}$. In this case, both $\Delta S_{E}[\bar{R}]$ and $\Delta E[\bar{R}]$ become unboundedly negative at large $\bar{R}$, so the family contains an escape path; since no member of the family has an action that exceeds $\bar{B}_{\mathrm{tw}}^{G}\left[\sigma_{\max }\right]$, Lemma 2 holds. By contrast, for the case

$\sigma_{\max } \geq\left(\sqrt{-V_{\mathrm{t}}}-\sqrt{-V_{\mathrm{f}}}\right) / \sqrt{6 \pi G}$ the family may not contain an escape path, but since $\bar{B}_{\mathrm{tw}}^{G}\left[\sigma_{\max }\right]=\infty$, Lemma 2 trivially holds. Thus we have proved Lemma 2 for all values of $\sigma_{\max }$.

As in the nongravitational case, this proof would still have gone through had we replaced $\phi_{\mathrm{t}}$ with any value in the range $\phi_{\mathrm{t}} \leq \phi_{\text {mid }}<\phi_{*}$, yielding the more powerful condition of Eq. (7).

[1] S. R. Coleman, The fate of the false vacuum. 1. Semiclassical theory, Phys. Rev. D 15, 2929 (1977); Erratum, Phys. Rev. D, 16, 1248(E) (1977).

[2] S. R. Coleman and F. De Luccia, Gravitational effects on and of vacuum decay, Phys. Rev. D 21, 3305 (1980).
[3] S. W. Hawking and I. G. Moss, Supercooled phase transitions in the very early universe, Phys. Lett. 110B, 35 (1982).

[4] D. A. Samuel and W. A. Hiscock, Effect of gravity on false vacuum decay rates for $\mathrm{O}(4)$ symmetric bubble nucleation, Phys. Rev. D 44, 3052 (1991). 
[5] H. Ooguri and C. Vafa, Non-supersymmetric AdS and the Swampland, arXiv:1610.01533; B. Freivogel and M. Kleban, Vacua Morghulis, arXiv:1610.04564; U. Danielsson and G. Dibitetto, Fate of stringy AdS vacua and the weak gravity conjecture, Phys. Rev. D 96, 026020 (2017); T. Banks, Note on a paper by Ooguri and Vafa, arXiv:1611.08953.

[6] S. R. Coleman, V. Glaser, and A. Martin, Action minima among solutions to a class of Euclidean scalar field equations, Commun. Math. Phys. 58, 211 (1978).

[7] T. Banks, C. M. Bender, and T. T. Wu, Coupled anharmonic oscillators. 1. Equal mass case, Phys. Rev. D 8, 3346 (1973).

[8] C. G. Callan, Jr. and S. R. Coleman, The fate of the false vacuum. 2. First quantum corrections, Phys. Rev. D 16, 1762 (1977).

[9] S. R. Coleman, Quantum tunneling and negative eigenvalues, Nucl. Phys. B298, 178 (1988).

[10] D. A. Samuel and W. A. Hiscock, 'Thin wall' approximations to vacuum decay rates, Phys. Lett. B 261, 251 (1991); H. Aoyama and S. Wada, Bounce in valley: Study of the extended structures from thick wall to thin wall vacuum bubbles, Phys. Lett. B 349, 279 (1995); I. Dasgupta, Estimating vacuum tunneling rates, Phys. Lett. B 394, 116 (1997); H. Widyan, A. Mukherjee, N. Panchapakesan, and R. P. Saxena, Bubble formation in $\phi^{4}$ theory in the thin wall limit and beyond, arXiv:hep-th/9803089; A. Masoumi, K. D. Olum, and B. Shlaer, Efficient numerical solution to vacuum decay with many fields, J. Cosmol. Astropart. Phys. 01 (2017) 051; A. Masoumi, K. D. Olum, and J. M. Wachter, Approximating tunneling rates in multi-dimensional field spaces, J. Cosmol. Astropart. Phys. 10 (2017) 022.
[11] G. Munster and S. Rotsch, Analytical calculation of the nucleation rate for first order phase transitions beyond the thin wall approximation, Eur. Phys. J. C 12, 161 (2000); G. V. Dunne and H. Min, Beyond the thin-wall approximation: Precise numerical computation of prefactors in false vacuum decay, Phys. Rev. D 72, 125004 (2005); R. V. Buniy, An iterative method for spherical bounces, arXiv:1610.00018.

[12] U. Gen and M. Sasaki, False vacuum decay with gravity in nonthin wall limit, Phys. Rev. D 61, 103508 (2000); Y. Goto and K. Okuyama, Numerical analysis of Colemande Luccia tunneling, Int. J. Mod. Phys. A 31, 1650131 (2016).

[13] A. Masoumi and E. J. Weinberg, Bounces with $\mathrm{O}(3)$ x $\mathrm{O}(2)$ symmetry, Phys. Rev. D 86, 104029 (2012); H. Lee and E. J. Weinberg, Negative modes of Coleman-De Luccia bounces, Phys. Rev. D 90, 124002 (2014); A. Masoumi, S. Paban, and E. J. Weinberg, Tunneling from a Minkowski vacuum to an AdS vacuum: A new thin-wall regime, Phys. Rev. D 94, 025023 (2016); S. Paban, E. J. Weinberg, and A. Masoumi, Generalized surface tension bounds in vacuum decay, Phys. Rev. D 97, 045017 (2018); K. Dutta, C. Hector, T. Konstandin, P. M. Vaudrevange, and A. Westphal, Validity of the kink approximation to the tunneling action, Phys. Rev. D 86, 123517 (2012).

[14] S. J. Parke, Gravity, the decay of the false vacuum and the new inflationary universe scenario, Phys. Lett. 121B, 313 (1983); J. D. Brown and C. Teitelboim, Neutralization of the cosmological constant by membrane creation, Nucl. Phys. B297, 787 (1988). 